\title{
Natural breakage of the very long intromittent organ of the seed bug Lygaeus simulans (Heteroptera: Lygaeidae)
}

\author{
LIAM R. DOUGHERTY and DAVID M. SHUKER
}

University of St Andrews - School of Biology, Sir Harold Mitchell Building Greenside Place, St Andrews, Fife KY16 9TH, U.K.; e-mails: liam.dougherty@uwa.edu.au;dms14@st-andrews.ac.uk

Key words. Heteroptera, Lygaeidae, Lygaeus simulans, genitalia, genital damage, breakage, mating plug

\begin{abstract}
The male intromittent organ of the seed bug Lygaeus simulans ends in a long, sclerotized structure which is used to transfer sperm during mating. Observations suggest that this structure becomes brittle and is liable to breakage after being artificially exposed to the air for an extended period of time. In this study we investigate the frequency of intromittent organ breakage in L. simulans. We first examined the intromittent organ of a sample of males that mated once, and found that breakage was rare. We hypothesised that breakages are likely to be more frequent if a male is able to mate multiple times, and so we next paired males with a female for 21 days in order to provide the opportunity for multiple mating. Almost a quarter $(22.5 \%)$ of these males exhibited signs of genital breakage. The point of breakage varied: for six males only the tip of the structure (around $6 \%$ of its length) was missing, whereas for three males over $50 \%$ of the structure was missing. However we were unable to locate any fragments of male genitalia in the reproductive tracts of any females that came into contact with these males. This suggests that breakages do not necessarily occur during mating itself, but instead probably occur as the intromittent organ is being retracted into the genital capsule following mating. In this species breakage may not significantly reduce male reproductive fitness as sperm transfer may still be possible.
\end{abstract}

\section{INTRODUCTION}

In many insect taxa the male intromittent organ ends in an elongate, sclerotized tube known as a flagellum or processus gonopori. This trait is seen for example in Coleoptera (Rodriguez, 1995; Gack \& Peschke, 2005; Matsumura \& Yoshizawa, 2010), Hemiptera (Deckert, 1990), Dermaptera (Kamimura, 2005; van Lieshout \& Elgar, 2011) and Neuroptera (Sziráki, 2002). In some species this structure is directly involved in sperm transfer (e.g. in Hemiptera: Deckert, 1990), however in others it may be used in conjunction with a spermatophore (Gack \& Peschke, 2005; Matsumura et al., 2014). The extreme length of these structures can make deployment and subsequent withdrawal following mating a complicated process. For example, in the rove beetle Aleochara tristis Gravenhorst the flagellum is more than twice the length of the male's body, and in order to prevent entanglement following mating the male holds the flagellum taut between the mesothorax and the pronotum whilst withdrawing it into the body, a behaviour known as "shouldering" (Gack \& Peschke, 2005).

The sclerotized nature of such structures means that they may also be very fragile and susceptible to breakages, either during intromission or during the deployment/ withdrawal process. This will be especially important if breakage significantly impairs a male's insemination ability, either because the intromittent organ is damaged so as to prevent sperm release, or because the length of the organ is reduced so that it is unable to release sperm in the correct place (e.g. Dougherty et al., 2015). Alternatively, such breakages could be adaptive if they increase a male's reproductive success. For example, in many spider species a portion of the male copulatory organ frequently breaks off inside the female reproductive tract during mating, and can act as a mating plug, preventing subsequent males from being able to inseminate the female (Snow et al., 2006; Uhl et al., 2010; Kuntner et al., 2015). In some species essentially all matings result in breakage (Uhl et al., 2010). Though breakage typically renders the copulatory organ non-functional, this strategy is likely favoured because males typically encounter few females and so have limited opportunities to mate (Uhl et al., 2010; Kuntner et al., 2015).

However, the susceptibility of elongate intromittent organs to breakage in insect species has been rarely tested. One exception is a series of studies on the earwig Euborellia plebeja Dohrn. In this species males possess a pair of extremely elongate intromittent organs known as virgae, which are able to remove rival sperm from the female reproductive tract (Kamimura, 2005). Males with broken virgae have been observed in the wild, though at a very low frequency, and breakages can be induced experimentally by interrupting matings (Kamimura, 2003). Experimental manipulations have shown that a female can be successfully inseminated even with a broken virga in the reproductive tract, suggesting that such breakages do not function as mating plugs (Kamimura, 2003). Breakages are likely a by-product on selection for extreme virga length, and the fact that males possess paired organs reduces the cost of breakage significantly (Kamimura \& Matsuo, 2001; Kamimura, 2003). In a more extreme case, male genital breakage has been shown to occur frequently following interspecific matings in the beetle Carabus maiyasanus Bates, caused by the poor fit between heterospecific male and female genitalia (Sota \& Kubota, 1998). These two ex- 
amples illustrate that non-adaptive genital breakages may occur in the wild in some species, however whether the frequency of breakage is high enough to influence genital evolution in these or other species is unclear.

In the seed bug Lygaeus simulans Deckert (Heteroptera: Lygaeoidea: Lygaeidae), the male intromittent organ ends in a coiled, sclerotized tube known as the processus gonopori (Ludwig 1926; Deckert, 1990), which is approximately $6.8 \mathrm{~mm}$ long (which is around $60 \%$ of the average male body length: Tadler, 1999; Dougherty et al., 2015). This structure is tightly coiled inside the male genital capsule prior to mating (Ludwig, 1926). During mating the entire intromittent organ is everted into the female bursa copulatrix, and the processus gonopori is then threaded along the female spermathecal duct (Gschwentner \& Tadler, 2000; Dougherty et al., 2015). The lumen of the spermathecal duct is narrower than the width of the processus gonopori (Gschwentner \& Tadler, 2000), so that any broken fragments of processus gonopori in the spermathecal duct from a previous mating will likely prevent subsequent males from inseminating the female.

Following mating in L. simulans the male must withdraw the entire intromittent organ back into the genital capsule. Males can be observed performing a characteristic 'handstand' behaviour, in which the hind legs are used to manipulate and straighten out the intromittent organ during withdrawal to avoid entanglement. In some cases withdrawal is not completed for several hours (L. Dougherty, per. obs.). Preliminary observations show that when the male intromittent organ is left exposed to the air for more than $24 \mathrm{~h}$ the processus gonopori becomes very brittle and can break easily. This structure may thus be more likely to break if a male fails to withdraw the intromittent organ properly into the genital capsule following a mating, or mates multiple times and so needs to perform multiple withdrawals. Such breakage could potentially also occur as the male and female genitalia are disengaged following mating, and indeed in the laboratory one of us has observed a female with a broken fragment of processus gonopori protruding from her reproductive tract (D. Shuker, pers. obs.). Importantly, it has been shown that artificial cutting of the processus gonopori does not impair sperm transfer, as the gonopore remains open (Dougherty et al., 2015). Thus the simple nature of the processus gonopori means that a 'clean' break may not render a male sterile, as long as there is a lumen through which seminal fluid may travel.

These observations thus beg the question: how often does such genital breakage occur in natural populations of L. simulans? Could breakage be frequent enough to significantly negatively impact male reproductive fitness in this species? We first examined the genitalia of a sample of males used in a previous experiment (Dougherty \& Shuker, in prep.) for signs of breakage. These males were all relatively young and mated only once. However, in the wild it is likely that both males and females may mate multiple times (note that the reproductive biology of $L$. simulans is very similar to L. equestris which has been well studied in this regard: Solbreck, 1972; Sillén-Tullberg, 1981). A potential way to estimate the upper limit of breakage frequency would be to allow males to mate many times across their lifespan. We therefore performed an experiment to assess the frequency of natural genital breakage in males allowed to mate freely for their entire lifetime.

\section{METHODS}

\section{Animal husbandry}

Lygaeus simulans is an aposematic, seed-feeding insect in the family Lygaeidae (though note that this family is paraphyletic; Henry, 1997; Burdfield-Steel \& Shuker, 2014a). The reproductive behaviour of both $L$. simulans and its sister species $L$. equestris L. has been well studied (Sillén-Tullberg, 1981; Shuker et al., 2006; Burdfield-Steel et al., 2013; Dougherty \& Shuker, 2014; Burdfield-Steel \& Shuker, 2014b). The study population originates from individuals collected in 2008 and 2009 from the Pratomagno region of Tuscany in Central Italy, and has been kept in the laboratory in continuous culture ever since. All individuals are kept in an incubator at $29^{\circ} \mathrm{C}$, with a $22 \mathrm{~L}: 2 \mathrm{D}$ cycle which prevents individuals from entering reproductive diapause. Prior to experiments individuals were removed from stock populations into small plastic deli tubs $(108 \times 82 \times 55 \mathrm{~mm})$ as nymphs. These tubs were checked every day for newly eclosed adults, which were then separated into single-sex tubs, with 8-10 individuals per tub. This ensures that all adults used in the experiments are virgins. All tubs were provisioned with de-husked sunflower seeds (Helianthus anuus) ad libitum as food, plastic tubes containing distilled water stoppered with cotton wool, and a piece of dry cotton wool as shelter. All experimental individuals were at least seven days old to ensure sexual maturity.

\section{Once-mated males}

This experiment was designed to measure the strength of phenotypic selection on processus gonopori length in L. simulans. All individuals in the experiment were $8-11$ day old virgins. A single male and female were placed into a small plastic Petri dish (55 $\mathrm{mm}$ diameter) at room temperature and under natural light, and observed for two hours. If a male did not mate after two hours he was euthanized by placing in $\mathrm{a}-13^{\circ} \mathrm{C}$ freezer. Mating pairs were checked every ten minutes for separation, after which the mated males were euthanized. A pair were said to be in copula when in the characteristic end-to-end mating position, with the intromittent organ properly inserted (the male may sometimes engage the external female genitalia but fail to insert the intromittent organ). If any pairs were still mating after eight hours they were manually separated by brushing with a fine paintbrush. This stimulates the male to detach from the female, and does not appear to cause any damage to the male genitalia (see below).

Following the experiment, the male processus gonopori was measured following Higgins et al. (2009). Briefly, the processus gonopori was removed and mounted on a microscope slide using double-sided sticky tape. The slides were then imaged using an Olympus SZX10 stereo microscope and attached camera, and measured using the image analysis program $\mathrm{Cell}^{\wedge} \mathrm{D}$ (Soft Imaging System, Olympus Corp). The processus gonopori was measured from the "turning point" (where the fleshy base of the intromittent organ ends) to the tip (Tadler, 1999; Dougherty et al., 2015). The processus gonopori ends in a characteristic cup-like structure which can be seen easily (see Fig. 2 in Dougherty et al., 2015). A male was said to exhibit a genital breakage if this tip was missing (Fig. 1). 


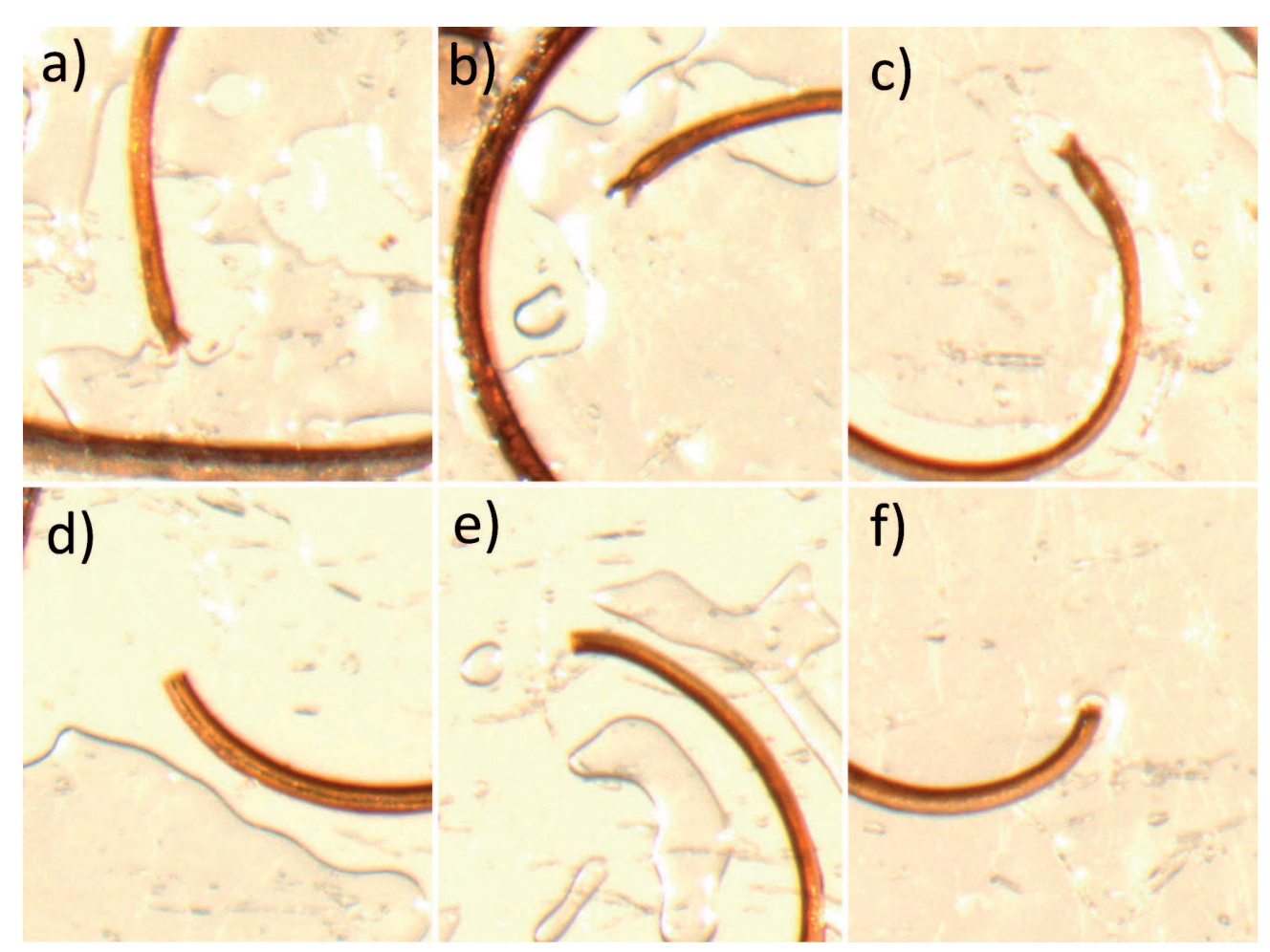

Fig. 1. Examples of removed male intromittent organs, with the top panels $(a-c)$ showing intact processus gonopori with tips visible, and the bottom panels (d-f) showing broken processus gonopori with no tips visible.

\section{Multiply-mated males}

We next performed an experiment to assess whether genital breakage was more frequent for males that were able to mate several times. All males were between 9 and 14 days old at the start of the experiment. A single virgin male was paired with a single virgin female in a small tub for 21 days, or until the male died. Forty males were used in total. Males and females were able to interact freely for the duration of the experiment. Pairs were checked three times a day at roughly four-hour intervals for copulation. Copulation duration in this species is highly variable, with a high proportion of matings lasting less than four hours (Micholitsch et al., 2000). Therefore such counts probably underestimate the number of intromissions that are actually performed by each male. We also cannot guarantee the mated status of males in this experiment the way we could in the previous experiment. Any females that died during the trial were replaced with another mature, virgin female. Adults were moved into new tubs each week if nymphs were present to prevent mould build-up. At the end of the trial any copulating pairs were manually separated (see above). All individuals were then euthanized by placing in $\mathrm{a}-13^{\circ} \mathrm{C}$ freezer, and the processus gonopori removed and was examined for signs of damage as described above. Additionally, the reproductive tract of any female that had come into contact with a male exhibiting a breakage was checked for the presence of any processus gonopori fragments (both in the bursa copulatrix and the spermathecal duct). All statistical analysis was performed in $\mathrm{R}$ version 3.0.1 (R Development Core Team, 2014). We used logistic regression to test how the likelihood of genital breakage was influenced by male age.

\section{RESULTS}

\section{Once-mated males}

Mating trials were performed with 140 males, of which 102 mated after two hours. A single mated male, and no un- mated males, exhibited signs of genital damage. The processus gonopori length of the broken male was $6.37 \mathrm{~mm}$. The average processus gonopori length for all males was $6.76 \mathrm{~mm}(N=140$, s.d. $=0.19 \mathrm{~mm})$.

\section{Multiply-mated males}

Nine males (22.5\%) exhibited genital breakage (of which three were never observed to mate). The average length of non-broken processus gonopori was $6.66 \mathrm{~mm}(N=31$, s.d. $=0.3 \mathrm{~mm}$ ). Of the nine males exhibiting a breakage, three were missing over $50 \%$ of the normal processus gonopori

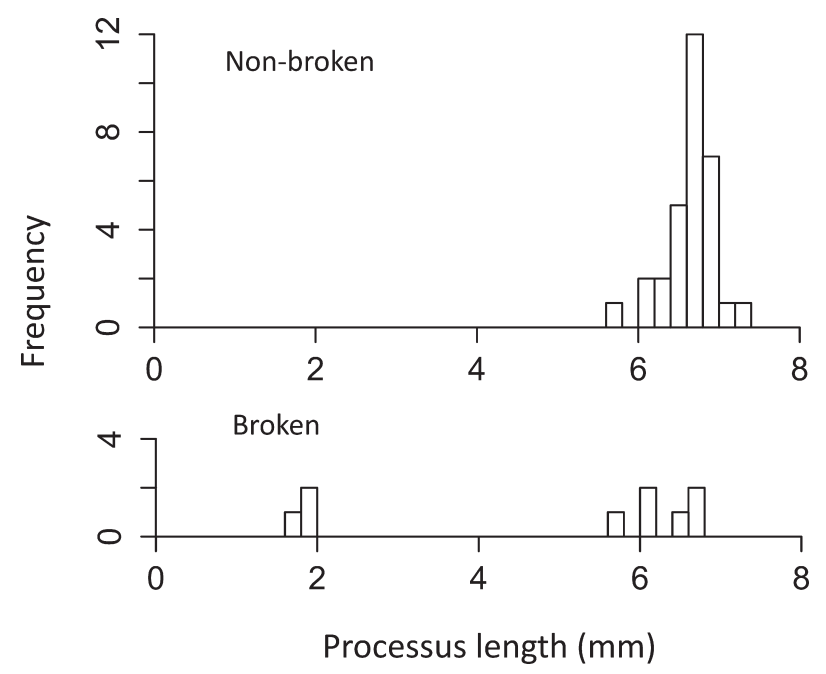

Fig. 2. Histogram showing the frequency distribution of processus gonopori length $(\mathrm{mm})$ for males possessing non-broken (top panel, $N=31$ ) and broken (bottom panel, $N=9$ ) intromittent organs. 


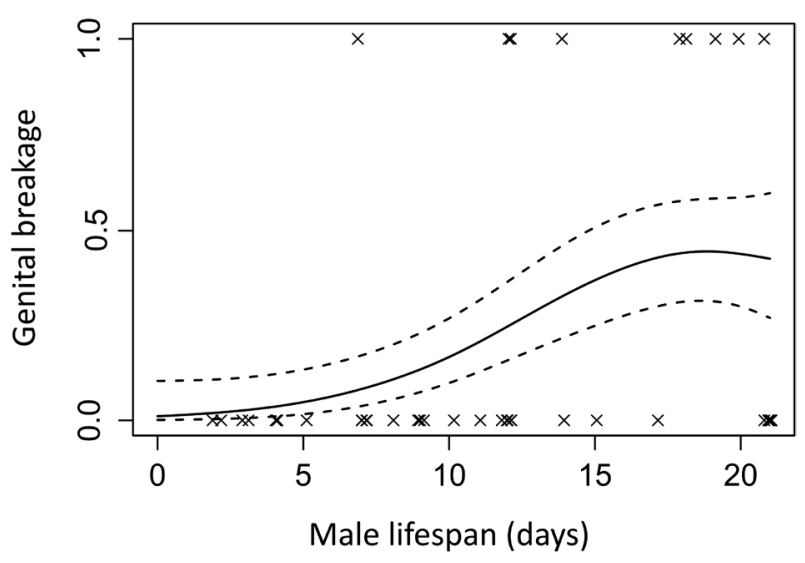

Fig. 3. The relationship between male lifespan (days since trial started) and the likelihood of exhibiting broken genitalia $(\mathrm{N}=9$ broken and 31 non-broken). The experiment was ended after 21 days.

length (Fig. 2). The average length of the processus gonopori of the remaining six broken males was $6.25 \mathrm{~mm}$ (s.d. $=0.42 \mathrm{~mm}$ ), which is a $6 \%$ reduction compared to intact males (see above). Importantly, we were unable to find any processus fragments in the reproductive tract of any female kept with the nine males exhibiting genital breakage.

Pairs were seen mating during $20 \%$ of observations. Eight males were never seen mating, however four of these lived less than ten days. There was no difference between broken and non-broken males in the proportion of observations they were seen mating (Mann-Whitney test: $\mathrm{U}=$ 137.5, $N=40, P=0.961$ ). Most males (34 out of 40 ) died before the end of the experiment: the average male lifespan was 11.58 days after the start of the trial (s.d.=6.16), with only six males surviving the full 21 days. The likelihood of genital damage increased with male lifespan (Logistic regression, $\chi_{1}^{2}=5.44, P=0.02$; Fig. 3).

\section{DISCUSSION}

By allowing males to mate many times during their lifetime, we have shown that a significant proportion of L. simulans males may suffer damage to their intromittent genitalia, specifically breakage of the long, fragile processus gonopori. This is a much higher rate of breakage than seen in young males that were only able to mate once, and it suggests that genital breakage may be ecologically relevant in this highly polygynous species. The fact that genital damage was not observed for any unmated males in the first experiment suggests firstly that damage does not occur prior to sexual maturity (or prior to our experiments), and that our method of separating pairs and preparing the processus gonopori for measurement does not lead to damage. Additionally, we were unable to find any fragments of male genitalia in the bursa copulatrix or spermathecal duct of females that came into contact with males exhibiting a breakage. This suggests that breakages do not necessarily occur during mating itself, or at least not while the processus gonopori is positioned along the spermathecal duct.

In the multiple-mating experiment, longer-lived males were more likely to exhibit genital breakage (Fig. 3). This suggests firstly that the breakages we have seen are not immediately deleterious to males. The processus gonopori consists of a single lumen which is used only for seminal transfer, so that damage does not lead to the loss of haemolymph (or other bodily fluids) and is unlikely to be a source of infection (Dougherty et al., 2015). However the average male lifespan in this experiment is much shorter than that observed in natural populations (Solbreck \& Sillén-Tullberg, 1990). We suggest this is because males in this experiment likely have many more opportunities to mate than they would in the wild mating, as mating interactions have been shown to reduce male and female longevity in the sister species L. equestris (Shuker et al., 2006). Reduced lifespan could also be the result of other factors such as unintended selection on shorter development times in laboratory culture. Longer-lived males may be more likely to have damaged genitalia because they will have performed more intromissions than shorter-lived males. Alternatively, the processus gonopori may become more brittle and liable to break in longer-lived males, irrespective of the number of intromissions. The experimental design used here makes it hard to distinguish between these explanations. We did not follow mating pairs continuously during the trial, and so the method used to record the frequency of copulation naturally underestimates the actual number of intromissions a male performs across his life, which would be the most important determining factor if breakage occurs during mating. Nevertheless, it seems likely that breakages are most likely to occur when the intromittent organ is outside the genital capsule following mating, with some rare exceptions (see introduction). This damage could be self-inflicted during withdrawal (as the male attempts to straighten out the intromittent organ with his back legs), or as a result of general environmental damage that occurs if withdrawal does not happen immediately.

We did not investigate the effect that genital breakage has on male reproductive fitness. The sclerotized nature of the processus gonopori means that damage to this structure does not result in the loss of haemolymph or a costly immune response. Additionally, males are probably unaware of any breakage as the structure is not innervated. For these reasons, male mating behaviour is unlikely to change following breakage. The most obvious effect that breakage would have on the males is their ability to successfully release sperm from the broken tip of the processus gonopori. Importantly, experimental ablation has shown that the distinctive tip of the intact processus gonopori - the structure that allows us to detect a breakage - does not play a key role in sperm release (Dougherty et al., 2015). Nevertheless, the processus gonopori could be rendered non-functional if the lumen is closed in some way so as to prevent sperm release. We presently have no data on how often a breakage results in the impairment of sperm release, though the structural properties of the processus gonopori suggest that it is not easily compressible. Furthermore, all breakages appear to be clean, in that they occur transversely at a $90^{\circ}$ angle. We therefore predict that natural breakages will not 
completely impair the sperm-transfer function of the processus gonopori.

Genital breakage could also indirectly influence male reproductive success due to the associated reduction in processus gonopori length. Both correlative and experimental studies on $L$. simulans have shown that male insemination success is lower for males with a shorter processus gonopori length (Tadler, 1999; Dougherty et al., 2015). For example, only 2 out of 28 mated males that had the processus gonopori shortened to an average of $4.84 \mathrm{~mm}$ (a reduction of approximately 29\%) succeeded in inseminating the female (Dougherty et al., 2015). Three of the males in the current study had a processus gonopori that was less than 2 $\mathrm{mm}$ long (a reduction of approximately 75\%; Fig. 2), and so it is very unlikely that insemination would be possible for these males. However, experimental shortening to an average of $6.48 \mathrm{~mm}$ (a reduction of approximately $5 \%$ ) led to males having fewer offspring following fertile matings, but did not influence the overall likelihood of insemination (Dougherty et al., 2015). Therefore a small reduction in length following a natural breakage, for example as seen for six of the males in this experiment (Fig. 2), may not impair male insemination success as long as sperm can still be released from the broken tip.

The fact that insemination may still be possible following genital breakage in L. simulans could suggest that the length of the processus gonopori could be in part driven by the likelihood of breakage (the "damage insurance" hypothesis). Previous studies in L. simulans have shown that the length of the male intromittent organ is subject to strong stabilising post-copulatory sexual selection, so that males with an intermediate processus gonopori length have the highest insemination success (Tadler, 1999). If small genital breakages (the breaking off of the tip of the processus gonopori) were common then longer-than-optimal processus gonopori lengths could potentially persist in the population, as a small reduction in length could actually increase male insemination success. However this hypothesis relies on the assumptions that breakage never significantly impairs sperm release, and that it typically occurs at the very tip of the processus gonopori. We cannot test this hypothesis without more data on the frequency of small breakages, and the ability of broken males to transfer sperm, in natural populations of $L$. simulans.

We found no evidence that broken male genitalia function as mating plugs. For example, in spiders adaptive genital breakage may be associated with structural weak points on the male copulatory organ which ensure that the same portion of the organ is broken off at each mating (Snow et al., 2006). The fact that the location of breakage is so variable in $L$. simulans thus suggests a non-adaptive function. Additionally, we did not find any processus gonopori fragments in the female reproductive tract that could prevent rival males from inseminating a female. The processus gonopori is a sclerotized structure that would be easily detected inside the transparent female reproductive tract. We thus suggest that the processus gonopori rarely breaks whilst inside the female. However one possibility we can- not rule out is that breakages do occur inside the female bursa copulatrix and then fragments of male genitalia are "flushed" out of the reproductive tract by the female during egg laying. However we note that a genital breakage in the bursa copulatrix would likely be ineffective as a mating plug anyway (due to its large size relative to the width of the processus gonopori: Dougherty et al., 2015), whether subsequently flushed by the female or not. The location of breakage in the female reproductive tract has been shown to strongly influence the efficacy of plugging in spiders (e.g. Snow et al., 2006).

In summary, we have shown that the distal, sclerotized part of the intromittent organ in L. simulans may frequently break in males that are able to mate multiple times. It is not clear exactly when breakages occur, though we suggest breakage is most likely as the intromittent organ is being withdrawn into the genital capsule following mating. It remains unclear to what extent breakages negatively influence male reproductive fitness, as breakage may not completely impair the insemination ability of males. In general, natural selection should oppose the evolution of genital structures that are liable to breakage, if such breakage reduced male reproductive success significantly. However such structures may persist in the population for example if mating is still possible following a breakage, or if breakage only occurs in older males that have already sired offspring. We suggest that more surveys of natural populations are needed to determine the impact that genital breakage has on male fitness in the wild, and that such (non-adaptive) breakages should be investigated for other insect species; especially those with elongate, fragile male genitalia.

ACKNOWLEDGEMENTS. We would like to thank the editor and three anonymous reviewers for their comments which improved the manuscript greatly, and the Natural Environment Research Council for funding.

\section{REFERENCES}

Burdfield-Steel E.R. \& Shuker D.M. 2014a: The evolutionary ecology of the Lygaeidae. - Ecol. Evol. 4: 2278-2301.

BurdField-STeEl E.R. \& Shuker D.M. 2014b: Mate-guarding in a promiscuous insect: species discrimination influences contextdependent behaviour. - Evol. Ecol. 28: 1031-1042.

Burdfield-Steel E.R., Dougherty L.R., Smith L.A., Collins L.A. \& Shuker D.M. 2013: Variation in social and sexual behaviour in four species of aposematic seed bugs (Hemiptera: Lygaeidae): the role of toxic and non-toxic food. - Behav. Process. 99: 52-61.

Deckert J. 1990: Zum Bau von Parameren, Phallus und Pygophore der Lygaeinae und Bemerkungen zur Systematik der Unterfamilie (Heteroptera, Lygaeidae). - Mitt. Mus. Naturk. Berl. 66: 91-119.

Dougherty L.R. \& ShuKer D.M. 2014: Pre-copulatory sexual selection in the seed bug Lygaeus equestris: a comparison of choice and no-choice paradigms. - Anim. Behav. 89: 207-214.

Dougherty L.R., Rahman I.A., Burdfield-Steel E., Greenway E.V. \& SHUKER D.M. 2015: Experimental reduction of intromittent organ length reduces male reproductive success in a bug. _ Proc. R. Soc. Lond. (B) 282: 20150724. 
Gack C. \& PeschKe K. 2005: 'Shouldering' exaggerated genitalia: a unique behavioural adaptation for the retraction of the elongate intromittent organ by the male rove beetle (Aleochara tristis Gravenhorst). — Biol. J. Linn. Soc. 84: 307-312.

Gschwentner R. \& TAdLer A. 2000: Functional anatomy of the spermatheca and its duct in the seed bug Lygaeus simulans (Heteroptera: Lygaeidae). — Eur. J. Entomol. 97: 305-312.

Henry T.J. 1997: Phylogenetic analysis of family groups within the infraorder Pentatomomorpha (Hemiptera: Heteroptera), with emphasis on the Lygaeoidea. - Ann. Entomol. Soc. Am. 90: $275-301$.

Higgins S.L., Hosken D.J. \& Wedell N. 2009: Phenotypic and genetic variation in male genitalia in the seedbug, Lygaeus equestris (Heteroptera). — Biol. J. Linn. Soc. 98: 400-405.

KamimuRA Y. 2003: Effects of broken male intromittent organs on the sperm storage capacity of female earwigs, Euborellia plebeja. - J. Ethol. 21: 29-35.

KAMIMURA Y. 2005: Last-male paternity of Euborellia plebeja, an earwig with elongated genitalia and sperm-removal behavior. - J. Ethol. 23: 35-41.

Kamimura Y. \& Matsuo Y. 2001: A "spare" compensates for the risk of destruction of the elongated penis of earwigs (Insecta: Dermaptera). — Naturwissenschaften 88: 468-471.

Kuntner M., Agnarsson I. \& Li D. 2015: The eunuch phenomenon: adaptive evolution of genital emasculation in sexually dimorphic spiders. - Biol. Rev. 90: 279-296.

LudwiG W. 1926: Untersuchungen über den Copulationsapparat der Baumwanzen. - Zoomorphology 5: 291-380.

Matsumura Y. \& Yoshizawa K. 2010: Insertion and withdrawal of extremely elongated genitalia: a simple mechanism with a highly modified morphology in the leaf beetle, Lema coronata. - Biol. J. Linn. Soc. 99: 512-520.

Matsumura Y., Yoshizawa K., Machida R., Mashimo Y., Dallai R., Gottardo M., Kleinteich T., Michels J., Gorb S.N. \& BeuTEL R.G. 2014: Two intromittent organs in Zorotypus caudelli (Insecta, Zoraptera): the paradoxical coexistence of an extremely long tube and a large spermatophore. - Biol. J. Linn. Soc. 112: 40-54.

Micholitsch T., Krugel P. \& Pass G. 2000: Insemination and fertilization in the seed bug Lygaeus simulans (Heteroptera: Lygaeidae). — Eur. J. Entomol. 97: 13-18.
R Development Core Team 2014: R: A Language and Environment for Statistical Computing. R Foundation for Statistical Computing, Vienna.

RodRIGUEZ V. 1995: Relation of flagellum length to reproductive success in male Chelymorpha alternans Boheman (Coleoptera: Chrysomelidae: Cassidinae). - Coleopt. Bull. 49: 201-205.

ShuKer D.M., Ballantyne G.A. \& Wedell N. 2006: Variation in the cost to females of the sexual conflict over mating in the seed bug, Lygaeus equestris. - Anim. Behav. 72: 313-321.

Sillén-Tullberg B. 1981: Prolonged copulation: A male 'postcopulatory' strategy in a promiscuous species, Lygaeus equestris (Heteroptera: Lygaeidae). - Behav. Ecol. Sociobiol. 9: 283-289.

Snow L.S., Abdel-Mesih A. \& AndRade M.C. 2006: Broken copulatory organs are low-cost adaptations to sperm competition in redback spiders. - Ethology 112: 379-389.

SolBRECK C. 1972: Sexual cycle, and changes in feeding activity and fat body size in relation to migration in Lygaeus equestris (L.) (Heteroptera., Lygaeidae). — Insect Syst. Evol. 3: 267274.

Solbreck C. \& Sillén-Tullberg B. 1990: Population dynamics of a seed feeding bug, Lygaeus equestris. 1. Habitat patch structure and spatial dynamics. - Oikos 58: 199-209.

Sota T. \& Кивота K. 1998: Genital lock-and-key as a selective agent against hybridization. - Evolution 52: 1507-1513.

SZIRÁKI G. 2002: Contribution to knowledge of female internal genitalia of Neuroptera. - Acta Zool. Acad. Sci. Hungar. 48: 341-349.

TADLER A. 1999: Selection of a conspicuous male genitalic trait in the seedbug Lygaeus simulans. - Proc. R. Soc. Lond. (B) 266: 1773-1777.

Uhl G., Nessler S.H. \& SCHNeIDER J.M. 2010: Securing paternity in spiders? A review on occurrence and effects of mating plugs and male genital mutilation. - Genetica 138: 75-104.

VAN Lieshout E. \& Elgar M.A. 2011: Longer exaggerated male genitalia confer defensive sperm-competitive benefits in an earwig. - Evol. Ecol. 25: 351-362.

Received July 14, 2015; revised and accepted September 9, 2015 Prepublished online September 22, 2015 